\title{
RESPUESTA A LOS COMENTARIOS DEL ARTÍCULO: EN CONTRA DEL POPULISMO REACCIONARIO: HACIA UNA NUEVA ARQUEOLOGÍA PÚBLICA
}

Es necesaria una aclaración inicial: el objeto de presentar esta versión en español de nuestro texto en Antiquity -pensada como una crítica esencialmente a la academia anglosajona- responde a dos objetivos: en primer lugar, debatir cuestiones que son de interés común con colegas latinoamericanos a quienes admiramos y que han realizado desde hace años una reflexión profunda sobre los temas que tratamos en nuestro artículo. Esta lógica situacional de nuestro texto, por responder a un reproche que Haber nos demanda, fue bien percibida por Tantaleán. En segundo lugar, el regreso del populismo reaccionario no es exclusivo del norte global. El final del ciclo progresista en América Latina y el auge de la extrema derecha (con Bolsonaro a la cabeza) indican que la crisis es compartida y la reflexión debe ser, también, compartida.

Una segunda aclaración, también necesaria, es que hablamos de ultraderecha. Efectivamente, como indica Acuto, Bolsonaro y Trump son la derecha nacionalista y la oligarquía de siempre, pero el lenguaje y los modos que utilizan son los del populismo chovinista-también el fascismo del siglo XX fue populista y oligárquico al mismo tiempo. Por eso utilizamos siempre el adjetivo "reaccionario". El ministro de finanzas de Bolsonaro es un neoliberal de escuela canónica. Pero su capacidad se multiplica en un juego político que no es el habitual de las élites conservadoras de siempre, sino que ha roto el espacio de la representación política moderna, construido a través del consenso liberal después de la Segunda Guerra Mundial (aquí señalar, por cierto, que el concepto que utilizamos de "liberalismo" es el mismo al que se refiere Gándara, y que, a pesar de la acepción inglesa tan cara en USA, no tiene nada que ver con la izquierda del espectro político), mediante la apelación directa al pueblo y su soberanía. A pesar de lo que dice Acuto, que pareciera querer salvar el populismo como opción posible para la resistencia, vemos necesario reconocer el fondo populista de estas estrategias. Esta colusión entre neoliberalismo y populismo reaccionario es algo más que anecdótico y nuestra sensación es que tenemos que parar mientes en ella, etnografiarla, analizarla en y a través de su expresión material, si es que queremos entender lo que nos ocurre. Y lo que nos ocurre es muy parecido en muchos países distintos. No es un privilegio del Norte o del Sur estar mejor, o peor, en esto. Las mismas tendencias se perciben en todas partes, desde Hungría a USA, desde Italia a Brasil, desde el Reino Unido a la India, o desde España a Argentina.
Lo que nos ocurre es una muestra del agotamiento de las soluciones liberales clásicas, a través de las cuales se impuso un modelo de modernidad individualista y el "siglo norteamericano", que es el siglo anglo-sajón. Y es también muestra de que la respuesta a este proyecto político desde la corrección política, las identity policies, y lo que Nancy Fraser resume en la estupenda etiqueta "neoliberalismo progresista", no solo está agotada sino que ha arrojado a todos los que sienten perdedores de esta parte de la historia, a votar masivamente opciones reaccionarias, que les perjudicarán. A Funari y Alarcón les parecen peligrosas nuestras críticas al cosmopolitismo y la tolerancia, e insisten en la importancia del conflicto en las relaciones humanas. No rechazamos esa visión; lo que criticamos es la visión liberal, neoliberal, de todo ello porque mistifica las relaciones de poder realmente existentes.

Esto es algo que también compartimos con Alejandro Haber, cuando apura la idea de que las comunidades son complejas y están atravesadas por antagonismos, pero no participamos en cambio de la disyuntiva que plantea: o la intervención se ciñe a los intereses comunitarios o se hace ciencia colonial. En primer lugar, creemos que es posible realizar arqueología en consonancia con el interés comunitario y al mismo tiempo que no esté circunscrita a la comunidad, es decir, que aborde problemas más amplios. Nada más lejos de nuestra intención que descartar a las comunidades, como interpretan Haber y Funari y Alarcón. Nuestro texto no aboga por reducir o desplazar el marco de acción política, sino por ampliarlo. Abogamos por una arqueología "que no se limite a los temas del patrimonio y de la relevancia local", no que deje de lado el patrimonio y lo local, como también pide Londoño. También abogamos por ampliar el espectro de la comunidad. Y nos preguntamos por qué nuestros colegas latinoamericanos no han dicho nada de los votantes de Bolsonaro o Macri, de los 20 millones de habitantes del Gran Buenos Aires o las periferias de Belo Horizonte o Bogotá.

Crear grandes narrativas críticas es parte del programa de arqueología pública que propugnamos. Haber ve en ellas mecanismos productores de colonialidad. Por esta regla de tres, las propuestas de los grandes teóricos de la decolonialidad -Quijano, Dussel, Grosfoguel- serían paradójicamente herramientas coloniales, dado que no se circunscriben a ninguna comunidad concreta y proponen grandes narrativas filosóficas, de aspiración universalista y explicativa ¿Es el pensamiento decolonial colonial? 
Acuto también sospecha de las grandes narrativas y cree que en vez de "salir a difundir compulsivamente y sin evaluar las narrativas que transmitimos", lo que tendríamos que hacer es ponernos al servicio de "los proyectos y luchas de los subordinados". No diremos que no. No vemos la contradicción aquí. Los relatos históricos que proponemos, no hace falta decirlo, deben ser reflexivos, pero además, ¿por qué van a ser incompatibles con los proyectos y luchas subalternos? ¿Es la gran narrativa que es El Capital incompatible con las aspiraciones de la clase obrera? Funari y Alarcón apuntan aquí algo en lo que no podemos estar más de acuerdo. Su comentario es un elogio de la historia y un recordatorio necesario de todo lo que la arqueología ha perdido por olvidarse del estudio de las condiciones de historicidad que constituyeron a nuestros objetos de estudio y constituyen hoy nuestro estudio. En España la arqueología ha estado tradicionalmente pegada a la historia (lo que dio lugar al problema contrario, contribuyendo a empobrecer la capacidad de acción de la arqueología como investigación histórica). Nosotros mantenemos la necesidad de volver a la historia para poder responder las preguntas de investigación que nos hacemos. No podríamos construir una cierta vuelta a las grandes narrativas sin ninguna referencia a la historia.

Angelo, en cambio, está de acuerdo en que las grandesnarrativas sonimportantes, perosugiereconstruir relatos que incorporen lo local, historias marginales y tradiciones de resistencia. Ese es, precisamente, el tipo de gran narrativa que estamos tratando de construir algunos (González-Ruibal 2014). También abogamos, naturalmente, por relatos cosmopolitas, como sugieren Funari y Alarcón. Nuestra crítica no es al cosmopolitismo como tal, sino al neoliberal y burgués. Defendemos un cosmopolitismo vernáculo, de los subalternos, que visibilice las solidaridades de los marginados. Si parece que queremos decir lo contrario, es que somos muy mal entendidos.

Igualmente resulta conflictiva nuestra reivindicación de la pedagogía, contra la que se rebelan Funari y Alarcón y Haber. Indudablemente hay muchas comunidades que no nos necesitan para interpretar el pasado, como recuerdan Funari y Alarcón y Tantaleán. Nunca ha sido nuestra intención, y así lo hacemos ver en el texto, extender nuestra propuesta a las comunidades indígenas, hacia las que, repetimos, la única actitud posible es de humilde aprendizaje y respeto. Pero vistos los resultados de las últimas elecciones en el mundo, parece que hay otros muchos colectivos que sí necesitan algún tipo de esfuerzo pedagógico por nuestra parte-como por ejemplo los 400.000 españoles que en Andalucía han depositado su confianza en un partido racista, machista y defensor a ultranza del Imperio Español. A ver si se nos entiende bien. A fuer de ser respetuosos, una parte básica del ethos del científico social que propugnamos, también respetamos a esta gente. No diremos que están engañados y que nosotros vamos a sacarlos del error. Diremos que tenemos que entender por qué votan cómo votan y eso supone para nosotros la obligación de analizar los discursos, en la práctica neo-coloniales y neo-imperiales, que los sitúan como objetivo y de analizar sus condiciones de producción y reproducción. Nos preocupa entender por qué estos discursos tienen más éxito en movilizar a la gente que los críticos que hacen las ciencias sociales, y ahí proponemos concentrar esfuerzo de investigación en los próximos años mientras, simultáneamente, ofrecemos nuestras capacidades pedagógicas para dar otras visiones que también dialoguen con los intereses de la gente. Obviamente sabemos, al menos desde Paulo Freire, que la enseñanza es una práctica transitiva, y que donde se enseña, también se aprende, algo que recuerda Angelo en su comentario. Pero más allá de esto, ¿es también colonial tratar de contrarrestar narrativas coloniales? Concordamos, de hecho, con el concepto de educación que ofrece Gándara: "como la formación de ciudadanos capaces de contribuir creativa y críticamente a la sociedad, transmitir lo mejor de su cultura actual a las siguientes generaciones, y transformar el mundo hacia algo más equitativo, sustentable y pleno". Ese debería ser el objetivo de la pedagogía arqueológica. Este énfasis en la educación no implica un desprecio del aprendizaje por nuestra parte. Existen numerosos modelos de pedagogía en los que aprender del otro es un pilar esencial (empezando por Paulo Freire, citado por Angelo) y a ellos nos remitimos. Pero también debemos ser conscientes de que es difícil y quizá no muy recomendable dialogar con un nazi. Es aquí donde entra el elemento de provocación, que también causa cierta perplejidad entre los comentaristas. No se trata, claro, de provocar a los colectivos sin tierra o a los desplazados en Colombia, sino al ciudadano biempensante que los desprecia o que ignora la historia de explotación y expropiación en que se fundamenta cualquier estado y cualquier imperio, responsable también de la existencia de los sin tierra o los desplazados.

Se advierte cierta discrepancia sobre si nuestra propuesta es objetivista o relativista. Para Haber nuestra defensa de la producción de un conocimiento objetivado y objetivable frente a la circulación de datos falsos y narrativas tramposas por parte de la ultraderecha implica que olvidamos el carácter situado de cualquier conocimiento, mientras que Gándara entiende que nuestra apelación al concepto de confianza Latouriano es demasiado posmoderno. La razón de esta discrepancia seguramente sea simple: nuestro modelo no se ajusta ni al objetivismo cientifista en el que nos quiere encasillar Haber ni al relativismo post-estructural. En el pasado los tres autores hemos adoptado como práctica posturas más simétricas. No es una pose. Es el reconocimiento de la necesidad de arriesgar en ejercicios que estén mestizados por los dos lados. Sabemos que la razón se constituye 
subjetivamente. Pero sabemos que la subjetividad no constituye la razón (Criado-Boado 2001); eso es así solo cuando se impone una subjetividad jerárquica preferente, que nosotros rechazamos. Para todo lo demás, creemos que debemos buscar una racionalidad objetivista postrelativista. Puede parecer un juego de palabras. Pero es un proyecto de investigación-acción. Alguno al menos de los autores, se siente bien con la capacidad deconstructiva de la modernidad burguesa por parte del pensamiento postmoderno radical (políticamente radical, no en el sentido de relativismo radical al que se refiere Gándara). Pero esto no es tan importante para este texto cuya posición intenta ser, como decimos, simétrica entre las diferentes dualidades que precisamente la Modernidad puso en juego. Creemos que los dilemas que nos plantea Gándara, aunque deben ser tenidos críticamente en juego, son perniciosos porque refuerzan un sistema de pensamiento diádico, occidental, cuyos efectos históricos son por ejemplo de sobra conocidos en América Latina por mor de la colonia. Para nosotros esas dualidades no constituyen opciones antagónicas por las que hubiera que decantarse, sino sistemas de racionalidad cruzada en la que nuestro pensamiento y acción se tienen que situar.

Otro punto conflictivo tiene que ver con la arqueología de contrato y comercial. Evidentemente, no defendemos el modelo capitalista de gestión del patrimonio, que es un desastre en todos los sentidos. La crítica a la arqueología de contrato, de hecho, tiende a olvidar el estatus precario de muchos proletarios de la profesión y la situación de privilegio de la que disfrutamos los que tenemos puestos permanentes en la academia (pero véase Zorzin 2015). Lo que insistimos es en la fragilidad del estatus de la arqueología y el patrimonio, del que puede prescindir el sistema en cualquier momento _ como recuerda Tantaleán para el caso de Perú. Dudamos que la arqueología de contrato sea un aliado efectivo del capitalismo, cuando el capitalismo corporativo hace tiempo que tiene poca necesidad de blanqueo ideológico. No vemos tampoco claro que las narrativas arqueológicas sean muy efectivas en la legitimación del neoliberalismo, como defiende Angelo, o, como escribe Londoño, que la arqueología siempre esté dispuesta a construir historias "del tamaño de las necesidades de quien las pueda pagar”. Quizá, pero en nuestra experiencia, nadie parece interesado en comprar arqueólogos para construir narrativas neoliberales o fascistas. Puede ser que esto ocurra en algún caso, pero parece que los think tanks conservadores están más interesados en comprar espacios de opinión en los medios y difundir sus posiciones económicas y sociales sobre temas más importantes para ellos, en los que realmente se juega la hegemonía en las guerras culturales. O quizá no encuentren suficientes arqueólogos dispuestos a ello, porque la realidad es que en España, Grecia, Polonia y Hungría son más bien periodistas y gente ajena a la academia la que está involucrada en la propaganda pseudohistórica de la ultraderecha. Es bastante frecuente que incurramos en análisis autocríticos lacerantes, en los que tememos vernos instrumentados por el poder o nos adelantamos al uso manipulativo que los "malos" puedan hacer de nuestros resultados. Pero en serio, creemos que tenemos que ser un poco más modestos. En realidad no importamos tanto a los poderosos. Más allá de esto, varios comentarios (por ejemplo Tantaleán, Angelo y Haber) concuerdan en la capacidad del patrimonio para vehicular estrategias situadas de resistencia y contrahegemónicas. Participamos de esta idea. Sabemos que puede ser porque el patrimonio tiene su propia fuerza para expresar otros sentidos e impone a veces su propio sentido. Celebrar una "minga", como recuerda Londoño, es siempre posible y bueno. Pero lo que no queremos es incurrir en ingenuidad. Que ello pueda ser así, no hace del patrimonio un arma revolucionaria. Nosotros preferimos una cierta cautela que se centra en ver que el patrimonio hoy en día ha sido subsumido enteramente en el funcionamiento del sistema de producción valor capitalista (lo que uno de nosotros llama "la Heritage machine", Alonso González 2018). Angelo hace muy bien cuando sitúa como trasfondo de la situación social del patrimonio en nuestras sociedades capitalistas el incendio del Museo de Río, o el capitalismo extractivista que generará unos efectos desbastadores en el área Andina en particular. Otros ejemplos palidecen cuando llegamos a estos dramas que tan a las claras muestran hasta qué punto el patrimonio y la cultura son mercancía capitalista y al mismo tiempo están desvalorizadas.

La acusación de colonialismo o colonialidad sobrevuela varios de los textos, de forma explícita o implícita. Concordamos en que es necesaria siempre la autocrítica y la reflexión sobre el lugar desde donde se enuncia. Por eso animamos a los autores a que se observen en el mismo espejo que han situado frente a nosotros, que evalúen sus posiciones de privilegio y se pregunten quién es su público, quiénes sus alumnos, cuál su estatus socioeconómico en sus respectivos países. Que se pregunten también porque ninguna mujer ha contribuido con sus comentarios a este debate ¿Cuáles son las desigualdades estructurales en la arqueología latinoamericana y española que hacen que hoy, aquí, no podamos escuchar sus palabras? También es sencillamente posible que tengan mejores cosas que hacer. Al margen de ello, debemos acabar reconociendo la honestidad intelectual y trabajo que los comentadores de este texto le han dedicado. La lista de temas que abren, de importantes puntos que consideran, es mucho más grande de lo que se puede replicar en un texto corto. En este sentido, nos consideramos afortunados por participar en este proceso de diálogo que extiende la motivación que declarábamos al principio. 


\section{Referencias Citadas} Alonso González, P. 2018. The Heritage Machine Fetishism
and Domination in Maragateria, Spain. Pluto Press, London.

Criado-Boado, F. 2001. Problems, functions and conditions of archaeological knowledge. Journal of Social Archaeology 1 (1):126-43.

González-Ruibal, A. 2014. An archaeology of Resistance. Materiality and Time in an African Borderland. Rowman \& Littlefield, Lanham.
Zorzin, N. 2015. Archaeology and capitalism: successful relationship or economic and ethical alienation? En Ethics and Archaeological Praxis, editado por C. Gnecco y D. Lippert, pp. 115-139. Springer, New York. 